Número 41 / Septiembre 2012

\title{
LA DISCAPACIDAD EN NUESTROS JÓVENES, ¿FOMENTA LAS RELACIONES NORMALIZADAS A TRAVÉS DE LAS REDES SOCIALES ONLINE O LES HACE ADICTOS A ELLAS?
}

\section{DISABILITY ON YOUNG PEOPLE: DOES IT ENCOURAGE NORMALIZED REATIONSHIPS THROUGH SOCIAL NETWORKS OR MAKE THEM ADDICTED TO THEM?}

\author{
Raquel Suriá Martínez \\ raquel.suria@ua.es \\ Universidad de Alicante
}

\section{RESUMEN}

En los últimos años, las redes sociales online se han convertido en un espacio de interacción social para muchos jóvenes. Sin embargo algunos usuarios pueden preferir estas redes para relacionarse. Este es el caso de multitud de jóvenes con discapacidad. En este trabajo se compara el perfil de uso y la opinión de los jóvenes con y sin discapacidad sobre las relaciones sociales que mantienen a través de estos espacios. Los resultados muestran mayor motivación en las relaciones virtuales en los jóvenes con discapacidad. Ello sugiere la importancia que tienen las redes online en sus relaciones sociales.

PALABRAS CLAVE: Adolescentes; discapacidad; internet; redes sociales; amigos; adicción.

\begin{abstract}
In recent year, online social networks have become an space of social interaction for many young people. However some users may prefer to relate to other people by using these networks. This is the case of many young people with disabilities. In this paper we compare the usage profile and the opinion of young people with and without disabilities about the friendly relations that they hold across networks. The results show a greater motivation in personal relationships in young people with disabilities. This suggests the importance of online networks in young people with disabilities.
\end{abstract}

KEYWORDS: Adolescents; disabled; internet; social networks; friends; addiction. 


\section{INTRODUCCIÓN}

En los últimos años se ha desarrollado un creciente interés por el estudio del impacto social de Internet y, en concreto, por la relación que jóvenes y adolescentes mantienen con este medio (Bernete, 2010; Buckingham, 2008; Fondevila, Carreras y del Olmo, 2012; Lenhart, Madden, Rankin Macgill y Smith, 2007; Livingstone, 2008; Rubio-Gil, 2010).

La facilidad para acceder a cualquier hora del día, los bajos costes, la eliminación de barreras, la respuesta rápida y las recompensas inmediatas hacen de estos espacios virtuales unos sitios muy atractivos que pueden hacer de este medio un espacio ideal para diferentes colectivos de jóvenes. Este puede ser el caso de los jóvenes con dicapacidad, puesto que les facilita la comunicación con otros usuarios sin tener las limitaciones de tener que desplazarse para interactuar con sus iguales y de este modo, poder compartir sus intereses, preocupaciones o necesidades, así como formar lazos, grupos, comunidades, etc, más fácilmente.

De este modo, la facilidad de conocer a otros usuarios de estos grupos sociales genera nuevos vínculos afectivos y permite a los jóvenes con limitaciones físicas cambios en su vida social que, de otra manera, podrían ser difíciles o incluso imposibles para ellos.

Es de sobra sabido, que establecer relaciones y explorar nuevas ideas o las funciones son en particular dos tareas cruciales para el desarrollo de los adolescentes (Erikson, 1963; Harter, 1999; Schaffer, 2002). Así, la literatura enfatiza la importancia de los efectos beneficiosos de las relaciones sociales en el bienestar individual a través de su influencia en el autoconcepto, autoestima, sentimientos de valía personal de los seres humanos (Arancibia, Herrera y Strasser, 1997; Jimenez y Pantoja, 2007; McKenna y Bargh, 1998; Niemz, Griffiths y Banyard, 2005).

Por tanto, es evidente que las redes sociales de internet pueden ser un vehículo para incrementar los lazos sociales y esto a su vez, puede ayudar a potenciar las habilidades sociales que va desarrollando la persona y con ello, mejorar las relaciones sociales tradicionales (Fowler y Christakis, 2009; McKenna y Bargh, 1999; Young, 2009; Tully, 2007; Zubeidat, Sierra y Salinas, 2007.

En relacióna esto, Morahan-Martin y Schumacher (2003), analizaron el efecto que podía tener la interacción a través de internet para disminuir la timidez y ansiedad social y así facilitar la formación de amistad en línea en varios grupos de estudiantes con distintos niveles de sentimientos de soledad. Para ello, crearon un foro e interactuaron durante varios meses. La soledad se evaluó mediante la Escala de Soledad UCLA (Russell, 1996). Todos los grupos redujeron sus sentimientos de soledad y potenciaron sus relaciones sociales fuera de la red.

Tal y como afirman Kraut et al, $(1998,2002)$, al estudiar a varios grupos de jóvenes con escasas habilidades sociales, la expansión que se consigue a través de ese medio virtual favorece los vínculos sociales que en un principio pueden ser escasos o inexistentes, llegando a integrarse en las vidas de estos jóvenes sin interferir en su vida real, y de esta manera, ayudarles a inrteraccionar en las relaciones cara a cara.

Por tanto, es fácil suponer como consecuencia de todo ello, que los jóvenes que padecen alguna discapacidad utilicen este recurso como forma de establecer relaciones con otros 
jóvenes, sin embargo, aunque sabemos que desde la incorporación de las tecnologías a nuestra sociedad multitud de jóvenes con discapacidad las han incorporado a su vida diaria, desconocemos el uso social que hacen de éstas, así, ¿se diferencian en el uso de las redes online al de los jóvenes sin discapacidad?, ¿se diferencian en el uso de las redes sociales virtuales que hacen estos jóvenes al de los usuarios de más edad?, ¿utilizan esta forma de comunicación para relacionarse con otros jóvenes?, ¿les ayuda a potenciar sus relaciones sociales tradicionales?, o a su vez ¿las utilizan para establecer nuevas amistades?, en otros términos, ¿han mejorado su vida social a través de estas redes?

Como se observa, el planteamiento de los estudios anteriores se basa en la convicción de que las redes sociales de internet poseen unas características peculiares que posibilitan un espacio social beneficioso para ciertos colectivos de usuarios (Berg y Peplau, 1982; Fowler y Christakis, 2008; Gross, Juvonen y Gable, 2002; Rapee y Heimberg, 1997; Williams y Solano, 1983), sin embargo, existen otras investigaciones que no son tan optimistas hacia este recurso.

En este sentido, uno de los riesgos más divulgado entre la literatura sobre el tema guarda una relación inversa con lo planteado hasta ahora. Por ejemplo, buena parte de los interesados en esta temática sugieren que el uso de las redes sociales virtuales puede facilitar el aislamiento, el bajo rendimiento, el desinterés por otros temas como las relaciones familiares y tradicionales (Bryant, Sanders-Jackson, y Smallwood, 2006; Cascales, Real y Marcos, 2011; Kraut et al, 1998; Morahan et al, 2000).

Como se ha mencionado en líneas anteriores, uno de los atractivos de internet para los jóvenes es que se caracteriza por la respuesta rápida, las recompensas inmediatas, la interactividad y las múltiples ventanas con diferentes actividades, aspectos que pueden convertirse aversivos cuando el abuso de la tecnología provoca aislamiento, induce ansiedad y le hace perder a la persona su capacidad de autocontrol (Cruzado, Matos y Kendall, 2006; Echeburua, 1999; Echeburúa y de Corral, 2009; García del Castillo et al, 2007; Niemz et al, 2005; Poch, 2009).

Con respecto a esto, recientemente otros autores han dirigido su atención hacia la propagación de trastornos como la depresión a través de estos espacios, enfatizando la capacidad de contagiar a través de la red este estado emocional entre los usuarios (Rosenquist, Fowler y Christakis, 2010).

En relación a esto, parece que hay ciertos colectivos más vulnerables que otros a las adicciones. Por ejemplo, los adolescentes constituyen un grupo de riesgo porque tienden a buscar sensaciones nuevas y son los que más se conectan a internet, además de estar más familiarizados con las nuevas tecnologías (Sánchez-Carbonell, Beranuy, Castellana, Chamorro y Oberst, 2008).

Otro grupo de riesgo puede ser el de las personas con enfermedades estigmatizantes (Hoybye, Johansen y Tjornhoj-Thomsen, 2005), problemas psicológicos (Estévez, Bayón, De la Cruz y Fernández-Liria, 2009; Yang, Choe, Balty y Lee, 2005) y personas con movilidad reducida o que padezcan alguna discapacidad (Alexander, Peterson y Hollingshead, 2003; Finn, 1999; Fogel, Albert, Schnabel, Ditkoff y Neugut, 2002). Con respecto a este último colectivo, el aislamiento social ha venido siendo una constante en la vida de las personas con discapacidad, por lo que, el hecho de que la persona no tenga fácil acceso a las relaciones tradicionales puede desembocar en un mayor uso y dependencia de las redes sociales virtuales (Berg y Peplau, 1982; McKenna y Bargh, 1998; Valkenburg, Peter y Schouten, 2006; Vitkus y Horowitz, 1987). 
Por tanto, de los estudios anteriores se desprende que ser joven y a su vez padecer una discapacidad pueden convertirse en factores motivadores hacia el uso de estas redes y con ello, de riesgo hacia el abuso de su utilización en los usuarios con estas características.

De forma que, centrando nuestro estudio en lo que los propios protagonistas piensan de su experiencia con el uso de estos espacios, es decir, la opinión de los jóvenes con discapacidad, ¿creen que pasan mucho tiempo conectados a las redes sociales de internet?, si esto es así, ¿piensan que interfiere el contacto con otros usuarios de estas redes en su vida cotidiana?,

Aunque el interés y por tanto, los estudios dirigidos a la utilización de las redes sociales entre los jóvenes es algo creciente actualmente, desconocemos los trabajos enfocados al uso y utilidad que hacen los jóvenes con discapacidad de estos espacios en sus relaciones sociales, por lo que, con el objetivo de acercarnos a profundizar en esta temática proponemos los siguientes objetivos:

- Conocer el perfil de uso que hacen los jóvenes con discapacidad de las redes sociales.

- Averiguar si este medio les ayuda a incrementar sus relaciones sociales.

- En este sentido y, con carácter comparativo, pretendemos conocer si existen diferencias en el uso de las redes sociales virtuales en función de padecer o no discapacidad, así como de ser joven adolescente o ser joven adulto. En concreto, esperamos que:

H1. Existan diferencias en el uso y satisfacción con este recurso entre los jóvenes con discapacidad y sin discapacidad.

H2. Los jóvenes de menos edad utilicen y les sea de más utilidad este medio para relacionarse.

- Finalmente, se pretende examinar si en algún grado, consideran estos jóvenes que el uso de las redes online repercute negativamente en su vida diaria.

\section{MÉTODO}

\section{Participantes}

En este estudio participaron 74 jóvenes (33 mujeres y 41 varones), con edades comprendidas entre los 18 y 35 años. De ellos, 42 padecían una discapacidad (56.8\%), (Tabla 1).

\begin{tabular}{clcc}
\hline \multicolumn{2}{c}{ Variables sociodemográficas } & N & $\%$ \\
\hline $\begin{array}{c}\text { ¿Padeces alguna } \\
\text { discapacidad? }\end{array}$ & No & 32 & 43,2 \\
Edad & Si & 42 & 56,8 \\
& Entre 18 y 25 & 45 & 60,8 \\
Género & Más de 25 & 29 & 39,2 \\
& Mujer & & \\
& Varó n & 33 & 44,6 \\
& & 41 & 55,4
\end{tabular}




\begin{tabular}{clcc} 
Formación académica & Estudios primarios & 18 & 24,3 \\
& Estudios secundarios & 28 & 37,8 \\
& Estudios universitarios & 28 & 37,8 \\
\hline Total & & 74 & 100,0 \\
\hline
\end{tabular}

Tabla 1. Perfil sociodemográfico

\section{Instrumentos}

El instrumento empleado ha sido un cuestionario elaborado y validado para el estudio, el cual está formado por tres bloques. El primero está relacionado con cuestiones sociodemográficas. El siguiente bloque guarda relación con preguntas para conocer el perfil de utilización y motivo se uso. La última parte del cuestionario se dedicó a conocer la frecuencia de uso de las redes online y los aspectos positivos y negativos que le encuentran a la utilización de las redes sociales. El instrumento consta de preguntas cerradas tipo si/no y de escala tipo Likert de cinco puntos, desde 1=nada a 5=mucho). Las propiedades psicométricas del cuestionario indicaron una consistencia interna del cuestionario se obtuvo a través del coeficiente Alfa de Cronbach, el cual indicó un $74 \%$ de fiabilidad, $(r=.74)$, por lo que se constata que esta escala posee una fiabilidad aceptable.

Para cumplir con los requisitos de validez de contenido se aplicó el protocolo de validación de contenido, que determina la relevancia o representatividad de los ítems en relación a la muestra establecida (Latiesa, 1996; Losada y López-Feal, 2003). Para ello, 2 jueces expertos respondieron a un cuestionario dicotómico que indagaba sobre la validez o no de cada ítem propuesto, a los resultados obtenidos se aplicó la Distribución Binomial para cada ítem, quedando el cuestionario compuesto por 22 ítems (ver Anexo).

En relación a la validez operativa los mismos expertos efectuaron recomendaciones en función de las cuales se ajustaron las preguntas. La información requerida buscaba opiniones sobre la claridad de las instrucciones y preguntas efectuadas a los participantes, los términos empleados para éste grupo, secuencia y número de ítems.

\section{Procedimiento}

Una vez diseñado el cuestionario, a través de la empresa "google" se creó un apartado específicamente para la línea de investigación a la que se accede a través de un enlace alojado en Google:

(https://spreadsheets.google.com/spreadsheet/embeddedform?formkey=dGZsSEZYa013VG 9hM1k0UEZ4ZHZZWGc6MQ). Para dar a conocer este enlace entramos en un espacio virtual dedicado al ámbito de la discapacidad, disponible en facebook destinado a temas de accesibilidad universal desarrollado por el observatorio de la discapacidad del Centro de apoyo al estudiante de la universidad de Alicante. A través de este espacio nos dirigimos a los participantes para explicarles brevemente nuestro objetivo, solicitando su colaboración para rellenar el cuestionario ubicado en el enlace inscrito en el mensaje. Posteriormente, se procedió al proceso de recogida de datos. La recopilación de los cuestionarios se llevó a cabo durante 2 meses.

De esta forma, se trata de una muestra no probabilística de jóvenes, con edades comprendidas entre los 18 y los 35 años (principales usuarios de estas redes según los datos existentes). De este modo, dividimos a los participantes en función de si padecían una discapacidad o no y en función de su edad (menores de 25 y mayores de esta edad). Establecimos los 25 años de edad de corte pensando en que es a partir de esa edad, en la 
que por término medio, un joven finaliza los estudios y se incorpora al mudo laboral y con ello, a su independencia y adultez).

\section{Análisis estadístico}

Para conocer el perfil de uso de las redes online de los jóvenes con discapacidad se hallaron las frecuencias, porcentajes y descriptivos de las variables sociodemográficas.

Para hallar las comparaciones entre los participantes en el uso y satisfacción de las redes sociales virtuales se utilizó la prueba $\chi^{2}$ en la variables categóricas.

Asimismo, se realizaron diversos análisis multivariados de covarianza (MANCOVA) para las variables independientes edad (menores de 25 vs, mayores de 25) y discapacidad (padecer discapacidad o no padecerla), con el género y el nivel de formación como covariantes. Se decidió controlar el efecto del género y del nivel de formación de los participantes para que las diferencias no se viesen afectadas por estas variables. Sin el control estadístico de estas covariantes, los efectos observados podrían explicarse, no por las diferencias entre los jóvenes en función de la edad o de padecer una discapacidad sino por el efecto de la interacción de estas covariables.

Después se realizaron ANCOVAs univariantes en los resultados significativos.

\section{RESULTADOS}

En nuestros resultados (Figura 1), observamos que el motivo principal por el que entran en las redes sociales los participantes es por amistad seguido de por estudio o trabajo, encontrándonos que los participantes con discapacidad indicaron entrar más por amistad (65.38\%), mientras que los participantes sin discapacidad mostraron que el motivo más frecuente era por estudio o trabajo $\left.(36.36 \%), \chi^{2}(4, N=74)=15.25, p<0.05\right)$.

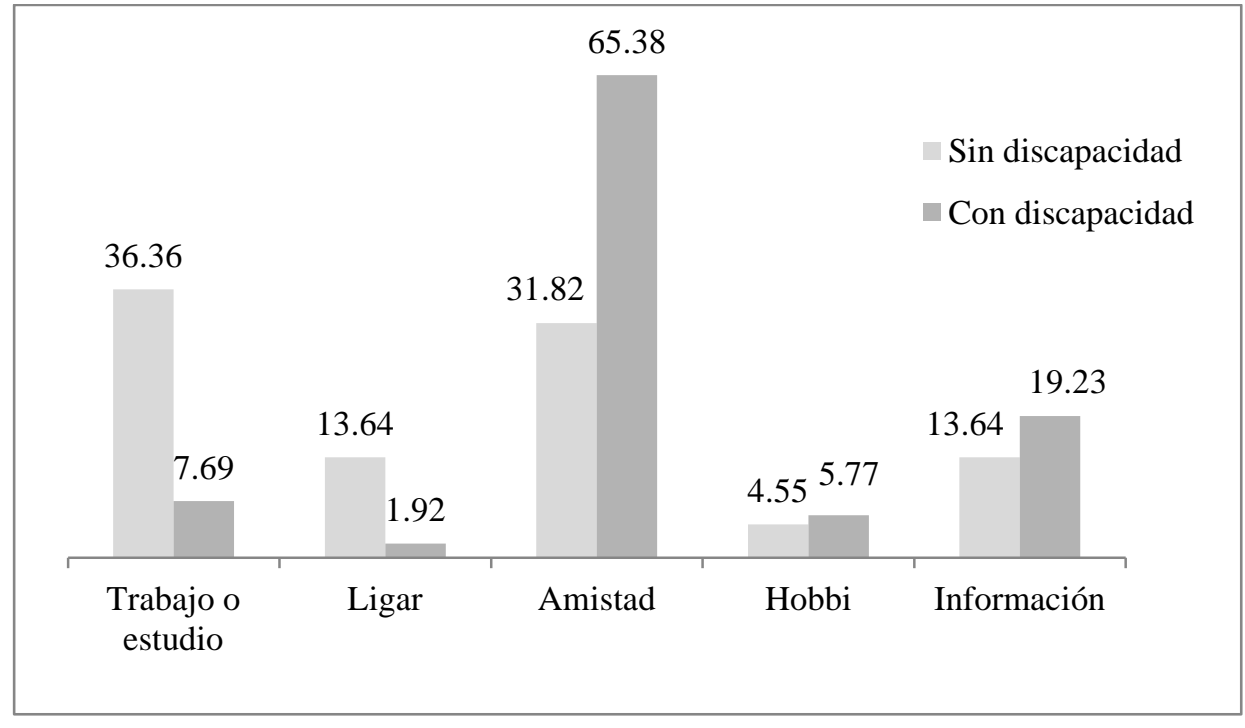

Figura 1. Motivo principal por el que entran en las redes sociales

Al examinar el motivo según el grupo de edad (Figura 2), se comprueba que los más jóvenes señalaron que la amistad era el motivo más frecuente por el que conectaban (62.22\%) mientras que los mayores de 25 años afirmaron entrar por buscar información (41.38\%), $\chi^{2}$ $(4, N=74)=13.9, p<0.05)$. 


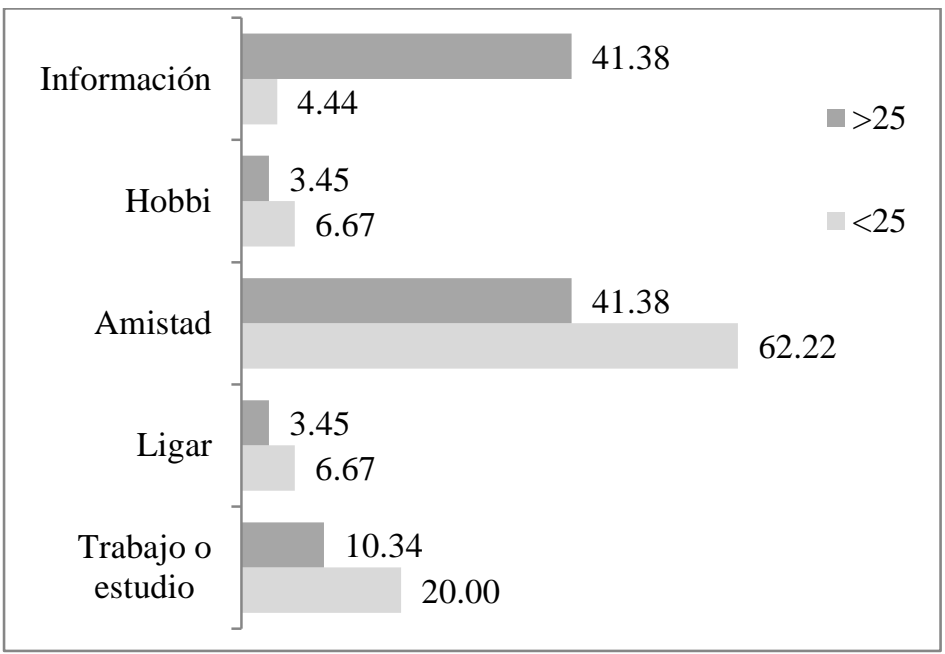

Figura 2. Motivo principal por el que entran en las redes sociales según edad

En referencia a los ítems de la escala que examinan el perfil de utilización (Tabla 2), podemos decir que en general, la puntuación media referente a: ¿Con qué frecuencia entras a alguna red social?, fue de 4.58 (D.T=1.31), puntuación que se encuentra entre 4=usar bastante y 5=mucho, las redes sociales. En el ítem relativo a: ¿Cuánto tiempo pasas en la red social?, encontramos la misma dirección, es decir, se encontraban entre pasar 4=bastante y $5=$ mucho tiempo en la redes, $(M=4.07, \mathrm{D} . \mathrm{T}=1.24)$.

Con respecto a la utilidad que encuentran en las redes online para relacionarse (Tabla 2), la gran mayoría conectaba ente $4=$ bastante y $5=$ mucho para reacionarse con amigos de la red $(M=4.15, D . T=0.84)$, seguidos de los que conectaban para relacionarse entre $3=$ algo $y$ 4=bastante con amigos tradicionales ( $M=3.47$, D. $T=1.22)$.

\begin{tabular}{|c|c|c|c|c|c|c|c|}
\hline Discapacidad & & $\begin{array}{l}\text { ¿Con qué } \\
\text { frecuencia } \\
\text { entras a } \\
\text { alguna red } \\
\text { social? }\end{array}$ & $\begin{array}{l}\text { ¿Cuánto } \\
\text { tiempo } \\
\text { pasas en la } \\
\text { red social? }\end{array}$ & $\begin{array}{l}\text { Utilizas las } \\
\text { redes para } \\
\text { relacionarte } \\
\text { con amigos } \\
\text { tradicionales }\end{array}$ & $\begin{array}{l}\text { Utilizas las } \\
\text { redes para } \\
\text { relacionarte } \\
\text { con amigos } \\
\text { de la red }\end{array}$ & $\begin{array}{c}\text { Utilizas las } \\
\text { redes para } \\
\text { relacionarte } \\
\text { con } \\
\text { compañeros }\end{array}$ & $\begin{array}{l}\text { Utilizas las } \\
\text { redes para } \\
\text { relacionarte } \\
\text { con } \\
\text { familiares } \\
\end{array}$ \\
\hline $\operatorname{Sin}$ & $M$ & 4.27 & 3.86 & 3.77 & 4 & 2.41 & 1.64 \\
\hline discapacidad & $D . T$ & 1.45 & 1.21 & 1.31 & 0.93 & 0.91 & 0.49 \\
\hline Con & $M$ & 4.71 & 4.15 & 3.35 & 4.21 & 2.35 & 1.54 \\
\hline discapacidad & $D . T$ & 1.24 & 1.26 & 1.17 & 0.8 & 0.93 & 0.5 \\
\hline \multirow{2}{*}{ Total } & $M$ & 4.58 & 4.07 & 3.47 & 4.15 & 2.36 & 1.57 \\
\hline & D.T & 1.31 & 1.24 & 1.22 & 0.84 & 0.92 & 0.5 \\
\hline$T$ & & 1.78 & 1.75 & 0.35 & 0.51 & 0.64 & 0.92 \\
\hline \multicolumn{8}{|l|}{ Edad } \\
\hline \multirow{2}{*}{$<25$} & $M$ & 5.36 & 4.53 & 3.09 & 4.2 & 2.47 & 1.49 \\
\hline & $D . T$ & 1 & 1.16 & 1.26 & 0.69 & 0.81 & 0.51 \\
\hline \multirow{2}{*}{$>25$} & $M$ & 3.38 & 3.34 & 4.07 & 4.07 & 2.21 & 1.69 \\
\hline & $D . T$ & 0.68 & 1.01 & 0.88 & 1.03 & 1.05 & 0.47 \\
\hline \multirow{2}{*}{ Total } & $M$ & 4.58 & 4.07 & 3.47 & 4.15 & 2.36 & 1.57 \\
\hline & D.T & 1.31 & 1.24 & 1.22 & 0.84 & 0.92 & 0.5 \\
\hline$T$ & & $88.86 * *$ & $30.18^{* *}$ & $11.87^{*}$ & 0.03 & $6.06 *$ & 3.2 \\
\hline
\end{tabular}

Tabla 2. Perfil de uso de las redes sociales

Al examinar si existen diferencias en el perfil de uso de las redes sociales en función de tener o no una discapacidad, los resultados del MANCOVA no fueron significativos (Lambda de Wilks $_{6,65}, F=0.63, p=.698$ ). Sin embargo, las covariables género (Lambda de Wilks $7,64, F=8.19$, 
$\mathrm{p}=.000$ ) y nivel de formación (Lambda de Wilks $7,64, F=11.59, p=.000$ ), indicaron influir en el perfil de utilización.

En referencia a la utilización de las redes sociales según la edad los resultados fueron significativos (Lambda de Wilks $6,65=28.05 \mathrm{p}=.000$ ). Del mismo modo, las covariantes género (Lambda de Wilks $6,65, F=11.40, p=.000$ ) y nivel de formación (Lambda de Wilks $6,65 F=13.63$, $\mathrm{p}=.000)$, también indicaron influir en los resultados.

Una vez controlado el efecto de estas covariantes, las dimensiones en las que encontramos diferencias estadísticamente significativas en función de la edad fue en la frecuencia de uso de las redes, $[F(1, N=74)=88.86, p=.000]$, en la que destacaron con una media más elevada los jóvenes de menos de 25 . También destacaron por pasar más tiempo en la red social, $[F(1$, $\mathrm{N}=74)=30.18, \mathrm{p}=.000]$, así como en la utilización para interaccionar con los compañeros, $[F(1$, $\mathrm{N}=74)=6.06, \mathrm{p}=.016]$. Por otro lado, fueron los más mayores los que destacaron por utilizar las redes sociales para conectar con los amigos tradicionales $[F(1, \mathrm{~N}=74)=11.87, \mathrm{p}=.001]$.

En relación a los ítems de la escala que examinan la opinión de los participantes sobre las redes sociales para fomentar o mejorar las amistades observamos (Tabla 3) que las puntuaciones medias se encuentran entre 3=estar algo de acuerdo y $4=$ bastante de acuerdo.

Así, en la Tabla 3, se presentan los valores medios obtenidos para cada uno de los ítems de la escala en función de tener o no una discapacidad. En primer lugar, el MANCOVA general fue significativo [Lambda de Wilks $\mathrm{K}_{6,65}$ ) $=10.04, \mathrm{p}=.000$ ], por lo que se procedió a realizar ANCOVAs univariantes para averiguar entre que ítems se encontraban las diferencias. Como puede comprobarse, excepto para los ítems relativos a ¿crees que en general, que las redes sociales te facilitan interaccionar con otros? y "en las redes virtuales tienes mejores amigos que en la vía tradicional", en los que no se encontraron diferencias a nivel estadísticamente significativo, fueron los jóvenes con discapacidad los que más de acuerdo estuvieron. También observamos un resultado significativo para la covariable formación (Lambda de Wilks $_{6,65}=2.81, p=.01$ ).

Tras controlar el efecto de esta covariable, es decir, del grado de formación observamos que los jóvenes con discapacidad estuvieron más de acuerdo que los jóvenes sin discapacidad en "Ia importancia del número de amigos virtuales que tienen", [F(1, N=74)=7.73, p=.05], también en el ítem referente a "estar de acuerdo en creer que las redes online mejoran las relaciones sociales", $[F(1, N=74)=24.84, p=.000]$ y finalmente en que "les han ayudado a mejorar sus relaciones sociales", $[F(1, \mathrm{~N}=74)=51.63, \mathrm{p}=.05]$.

Con respecto la opinión de los participantes sobre las redes sociales para fomentar o mejorar las amistades en función de la edad, el análisis arrojó diferencias estadísticamente significativas para esta variable, (Lambda de Wilks $_{6,65}=3.16, p=.01$ ), así como para la covariante nivel de formación, (Lambda de Wilks $6,65=2.92, p=.01$ ).

Al examinar los ítems en los que existían diferencias únicamente fue el referente a: ¿te han ayudado a mejorar tus relaciones sociales?, $[F(1, N=74)=15.08, p=.05]$, en el que destacaron los participantes de menos de 25 años por tener medias más elevadas que los de más edad.

\begin{tabular}{cccccc}
\hline & ¿Es & ¿Crees & ¿Te han & ¿Crees que son & En las redes \\
importante & que & ayudado a & verdaderas las & tienes mejores \\
Discapacidad & el número & mejoran & mejorar tus & amistades & amigos que vía \\
& de amigos & las & relaciones & online ? & tradicional \\
& que tienes & relaciones & sociales? & & \\
\hline
\end{tabular}




\begin{tabular}{ccccccc}
\hline Sin & $M$ & 3,14 & 2,64 & 2.50 & 4.41 & 1.77 \\
discapacidad & $D . T$ & 0.94 & 0.49 & 0.51 & 0.85 & 0.43 \\
Con & $M$ & 3.75 & 3.67 & 3.79 & 3.96 & 2.17 \\
discapacidad & $D . T$ & 0.84 & 0.88 & 0.75 & 0.82 & 0.88 \\
Total & $M$ & 3.57 & 3.36 & 3.41 & 4.09 & 2.05 \\
& $D . T$ & 0.91 & 0.92 & 0.91 & 0.85 & 0.79 \\
\hline$T$ & & $7.73^{*}$ & $24.84^{* *}$ & $51.63^{* *}$ & 2.51 & 2.34 \\
\hline Edad & & & & & & \\
& & & & & 3.91 & 2.07 \\
& & 3.6 & 3.6 & 3.71 & 0.85 & 0.89 \\
& $D . T$ & 0.84 & 1.01 & 0.92 & 4.08 & 2.03 \\
& $M$ & 3.52 & 3.3 & 3.43 & 0.78 & 0.63 \\
Total & $D . T$ & 1.02 & 0.6 & 0.65 & 4.09 & 2.05 \\
& $M$ & 3.57 & 3.36 & 3.41 & 0.85 & 0.79 \\
\hline \multirow{2}{*}{$t$} & $D . T$ & 0.91 & 0.92 & 0.91 & 4.12 & 0.04 \\
\hline
\end{tabular}

Tabla 3. Utilidad de las redes sociales para fomentar o mejorar las amistades

Finalmente, la opinión que mostraron sobre las repercusiones negativas que encuentran en las redes online las puntuaciones se encuentran entre 1=nada de acuerdo y $2=$ poco de acuerdo.

Así, al examinar las medias de las repercusiones negativas con el uso de las redes sociales no encontramos diferencias ni en función de tener discapacidad (Lambda de Wilks $2,69=1.50$, $\mathrm{p}=.229$ ), ni en función de la edad (Lambda de Wilks ${ }_{2,69}=1.84, \mathrm{p}=.832$ ) en ninguno de los dos ítems referidos a este aspecto.

\begin{tabular}{cccc}
\hline Discapacidad & $\begin{array}{l}\text { ¿Crees que desde que entras en las } \\
\text { redes de internet te has aislado de las } \\
\text { amistades tradicionales? }\end{array}$ & $\begin{array}{l}\text { ¿Crees que las redes de internet } \\
\text { interfieren negativamente en tu } \\
\text { vida? }\end{array}$ \\
\hline Sin & $M$ & 1,72 & 1.68 \\
discapacidad & $D . T$ & 0,45 & 0.47 \\
Con & $M$ & 1,55 & 1.55 \\
discapacidad & $D . T$ & 0.67 & 0.50 \\
Total & $M$ & 1.60 & 1.9 \\
& $D . T$ & 0.61 & 0.49 \\
\hline$T$ & & 0.28 & 0.32 \\
\hline Edad & & & 1.62 \\
& & 1.6 & 0.49 \\
& $M$ & 0.65 & 1.55 \\
& $D . T$ & 1.62 & 0.5 \\
& $M$ & 0.56 & 1.59 \\
Total & $D . T$ & 1.60 & 0.49 \\
\hline \multirow{2}{*}{$t$} & $M$ & 0.61 & 0.59 \\
\hline
\end{tabular}

Tabla 4. Repercusiones que encuentran con el uso de las redes sociales

\section{DISCUSIÓN}

En este trabajo se pretende reflejar la importancia que tiene para los jóvenes con discapacidad las redes sociales online en sus relaciones sociales. Así, tal y como se comprueba en nuestros resultados, podemos observar que este recurso es utilizado por la mayoría de jóvenes, y no tan jóvenes, que padecen una discapacidad, frecuentando gran parte de ellos estos espacios con asiduidad y dedicándole bastante tiempo al día.

Por tanto, aunque es evidente que estos espacios son utilizados por usuarios sin ningún tipo de dispapacidad, para algunos jóvenes con características específicas, como padecer una 
discapacidad, se hace un recurso especialmente útil que va a aportarles la posibilidad de reducir sus limitaciones e incrementar las vías de interacción.

En este sentido, los resultados han reflejado que los motivos específicos de las redes para la mayoría de jóvenes con discapacidad, suele ser para relacionarse con otros. Del mismo modo, los jóvenes que no padecen esta problemática son bastante proclives a entrar en las redes para conectar con sus amistades, aunque parece que estos últimos, tienen cierta tendencia a conectar más con amigos de la vida real, mientras que los jóvenes con discapacidad tienden a conectar con amigos conocidos a través de la red.

Esto evidencia que las redes sociales de internet ofrecen a los jóvenes nuevos contextos de relación social e interacción personal y ser un vehículo para incrementar los lazos sociales (Raacke y Bonde-Raacke, 2008; Magnuson y Dundes, 2008; Zywica y Danowski, 2008).

No obstante, cabe mencionar que el hecho de pasar más tiempo dentro de las redes sociales, no significa que las personas aumenten su interacción social, ni que obtengan mayor satisfacción en sus relaciones, pues el elemento esencial de la socialización y del proceso de comunicación se encuentra en la calidad de las interacciones de los jóvenes (Rivero, 2005).

De acuerdo con esto y aunque estos espacios virtuales incrementan las relaciones sociales, este tipo de relaciones son denominadas por algunos autores como relaciones "débiles" (Adelman, Parks y Albrecht, 1987; Mesch y Talmud, 2006). Así, Granovetter (1973) fue el primero en definir a las relaciones de "lazo débil" como el tipo de conexión que se establece entre individuos que se comunican diariamente pero que no son íntimas.

Sin embargo, al examinar la opinión de los participantes de nuestro estudio sobre las relaciones de amistad que mantienen en la red, observamos que esto parece no ser cierto ya que, las relaciones que matienen virtualmente, las consideran y valoran como algo importante y verdadero.

En este sentido, algunos expertos afirman que las redes online pueden promover la relación social por su naturaleza universal; de algún modo, se crea un espacio de relación donde la comunicación no está condicionada por la ubicación geográfica, la discapacidad, etc. (Ando y Sakamoto, 2008; Thayer y Ray, 2006; Valkenburg y Peter, 2008). Este tipo de relaciones sirve como un atajo para conocer a alguien, evitando el tiempo y el esfuerzo que implica una interacción cara a cara (Lea y Spears, 1995).

En relación con esto, hemos mencionado que las personas con discapacidad tienen mayor riesgo de tener un nivel de interacción social "tradicional" más reducido como consecuencia de las limitaciones fisicas (movilidad reducida, estigma, etc.), por lo que, es posible que el miedo a las señales sociales pueda reducir mucho su círculo de relaciones. Sin embargo en la red, tendrían una forma fácil de evitar esa sensación, el anonimato visual de 10 s comunicadores y la carencia de la presencia de los participantes y el encuentro social repetido a través de la red aumentarían las posibilidades de la interacción aliviando la sensación de ansiedad y con ello, desarrollar sus habilidades sociales (Finn y Lavitt, 1994; King y Poulos, 1998; Madara, Kalafat y Miller, 1988).

Como se comprueba en nuestro estudio, los jóvenes indicaron mejorar sus relaciones al interaccionar con otros a través de las redes, de modo que, estos recursos pueden ayudar a potenciar las habilidades sociales que va desarrollando la persona y mejorar las relaciones sociales tradicionales. 
Otro de los ejes en el que nos hemos centrado ha sido en comprobar si la edad afecta al patrón y motivo de utilización, comprobando un perfil diferente en nuestros participantes de menor y mayor edad, así, encontramos que los más mayores entran menos tiempo y cuando conectan, lo hacen para interactuar más con amigos tradicionales.

Esto indica que los jóvenes de más edad tienen su círculo o red de amigos tradicionales más establecida que los más jóvenes, pudiendo utilizar estos espacios únicamente para quedar, compartir comentarios o ver fotos de sus amigos.

Del mismo modo, observamos que los más jóvenes frecuentemente, conectan más para relacionarse con los compañeros que los más mayores. Esto, en cierta medida es comprensible si suponemos que el grupo de menos edad, se sitúa entre la población que está estudiando y por ello, tener más posibilidad de relacionarse con sus compañeros del instituto o universidad a través de las redes sociales.

Si indagamos en la importancia que tienen las relaciones virtuales con las que se relacionan a través de estos espacios encontramos que tanto los adolescentes como los jóvenes de mayor edad valoran considerablemente estas amistades, creen que mejoran las relaciones sociales e incluso indican que a ellos les ha ayudado a mejorar su círculo de amistades. Asimismo, se comprueba que tanto los adolescentes como jóvenes de más edad confían más en estos espacios para mejorar las relaciones sociales e incluso se sienten más ayudados a mejorar sus relaciones.

Estos resultados están en línea con los obtenidos por McKenna y Bargh, (1999), que entrevistaron a un total de 600 jóvenes usuarios de internet, el $67 \%$ contestó que su círculo social había aumentado y apenas el $4 \%$ informó de que tenían menos conocidos y amigos como resultado de usar este medio.

Como se mencionó, la expansión que se consigue a través de ese medio virtual favorece los vínculos sociales llegando a integrarse en las vidas de estos jóvenes sin interferir en su vida real, y de esta manera, ayudarles a inrteraccionar en las relaciones cara a cara (Katz y Aspden, 1997; Patterson et al, 1998, 2002; Selwyn, 2003).

Ásimismo y al igual que otros autores han hallado, nuestro trabajo indica que existen otras variables como el género (Magnuson y Dundes, 2008; Mesch y Talmud, 2006) y el nivel de formación (Valkenburg y Peter, 2008), que afectan a nuestros resultados; sin embargo, gracias a las pruebas estadísticas utilizadas hemos podido controlar su influencia y por ello, excluir su estudio en el presente trabajo.

Finalmente, no parece que existan opiniones contrarias a los beneficios de estas redes ni en función de padecer una discapacidad o no, ni entre ser adolescente o ser más mayor.

Como multitud de estudios revelan, Cruzado et al., 2004, Graner, Castellana, SanchezCarbonell, Beranuy y Chamarro, 2006; Morahan-Martin y Schumcher, 2000) determinadas características sociodemograficas como la edad, o personales (problemas, físicos y psicológicos), y psicosociales podrían actuar como factores predisponentes para el uso y/o abuso de Internet, pues este medio de comunicación virtual puede convertirse en un sustitutivo de otras relaciones personales directas para personas con déficits en habilidades sociales, timidez o complejos (Echeburua, 1999; Figuer, González, Malo y Casas, 2005). Sin embargo, que sean factores de riesgo no nos lleva a presuponer que los jóvenes con alguna de estas características utilicen con exceso este recurso. 
En relación a esto, McKenna y Bargh (1999), analizaron los aspectos sociales y de la personalidad en los procesos de interacción en la red, concluyendo que las personas tienen diferentes motivaciones y razones para usar internet, y que su uso diferencial provoca consecuencias diferentes, no pudiendo, por tanto, especificarse el efecto directo que puede tener sobre el individuo el uso de internet.

En cualquier caso, nuestro estudio examina el perfil de uso, las aportaciones y las repercusiones que creen los participantes que tienen con la utilización de las redes sociales, no analizando en este caso de forma objetiva, si realmente repercute negativamente en sus vidas.

\section{Limitaciones del estudio}

En el presente estudio nos hemos interesado principalmente en conocer si las redes sociales ayudan a mejorar y potenciar las amistades de los jóvenes con discapacidad, sugiriéndonos los resultados efectos beneficiosos con el uso de estos espacios.

Sin embargo, nuestro trabajo presenta algunas limitaciones que es necesario tener en cuenta.

Así, la primera hace referencia a la muestra, el número de participantes es muy reducido. Tampoco hubo una estimación de cálculo de tamaño de muestra, puesto que el muestreo no fue probabilístico, sino por conveniencia. En este sentido, se utilizó el muestreo "bola de nieve", así, inicialmente se colocó el enlace en una dirección de una red social, concretamente en un espacio relacionado con la discapacidad perteneciente a Facebook, transcendiendo el enlace a usuarios que, aunque eran conocidos por los usuarios con los que contactamos en un principio, desconocemos sus características.

Del mismo modo, la muestra de jóvenes sin discapacidad que colaboraron respondiendo al cuestionario accedieron a este espacio relacionado con la discapacidad, siendo posible que tengan alguna vinculación con el ámbito de la discapacidad, y por tanto, no representen en general, a los jóvenes sin discapacidad.

Igualmente, debemos ser cautos y considerar algunas limitaciones de este estudio relacionadas con el instrumento para recoger la información y con la deseabilidad social. Así, nuestros participantes son usuarios y al realizar un autoinforme sobre las cuestiones referidas en el cuestionario pueden estar sesgando sus respuestas.

Finalmente, nos hemos dirigido a los jóvenes con discapacidad, no pudiendo determinar el tipo de discapacidad que padecen los participantes, presuponemos que esta variable es muy relevante de análisis.

Por tanto, sería interesante superar estas limitaciones y ampliar el estudio a una muestra más elevada y representativa tanto para los jóvenes con discapacidad como para los jóivenes que no padecen esta problemática.

\section{REFERENCIAS}

Adelman, M., Parks, M. y Albrecht, T. (1987). Beyond close relationships: Support in weak ties. En T. L. Albrecht y M. B. Adelman (Eds.), Communicating Social Support (pp.126147). Newbury Park, CA: Sage. 
Alexander, S. C., Peterson, J. L. y Hollingshead, A. B. (2003). Help is at your keyboard: support groups on the Internet. En L. R. Frey (Ed.), Group communication in context: Studies of bona fide groups (pp. 309-334). Mahwah, NJ: Erlbaum.

Ando, R. y Sakamoto, A. (2008). The effect of cyber-friends on loneliness and social anxiety: Differences between high and low self-evaluated physical attractiveness groups. Computers in Human Behavior, 24, 993-1009.

Arancibia, V., Herrera, P. y Strasser, K. (1997). Manual de Psicología Educacional. Santiago de Chile: Universidad Católica de Chile.

Berg, J. y Peplau, L. (1982). Loneliness: the relationship of self-disclosure and androgyny. Personality and Social Psychology Bulletin, 8, 624-630.

Bernete, F. (2010). Usos de las TIC, relaciones sociales y cambios en la socialización de las y los jóvenes. Revista de estudios de juventud, 88, 97-114.

Bryant, J.A, Sanders-Jackson, A. y Smallwood, A.M. (2006). IMing, text messaging, and adolescent social networks. Journal of Computer-Mediated Communication, 11, 577-592.

Buckingham, D. (2008). Más allá de la tecnología. Aprendizaje infantil en la era de la cultura digital. Buenos Aires: Manantial.

Cascales, A., Real, J.J. y Marcos, B. (2011). Las redes sociales en internet. EDUTEC, Revista Electrónica de Tecnología Educativa, 38. Recuperado el 02/01/2012. En: http://edutec.rediris.es/Revelec2/Revelec38/redes_sociales_internet.html

Cruzado, L., Matos, L. y Kendall, R. (2006). Adicción a Internet: Perfil clínico y epidemiológico de pacientes hospitalizados en un instituto nacional de salud mental. Revista Medica Herediana, 17, 196-205.

Echeburúa, E. (1999). ¿Adicciones sin drogas? Las nuevas adicciones. Bilbao: Desclée de Brouwer.

Echeburúa, E. y Corral, P. (2009). Las adicciones con o sin droga: una patología de la libertad. En E. Echeburúa, F.J. Labrador y E. Becoña (eds.), Adicción a las nuevas tecnologías en adolescentes y jóvenes (pp. 29-44). Madrid: Pirámide.

Erikson, E. (1963). Childhood and society. New York: Norton. Nueva York: Norton.

García del Castillo, J.A., Tero, M.C., Nieto, M., Lledó, A., Sánchez, S., Martín-Aragón, M. y Sitges, E. (2008). Uso y abuso de Internet en jóvenes universitarios. Adicciones, 20, 131-142.

Estévez, L., Bayón, C., De la Cruz, J. y Fernández-Liria, A. (2009). Uso y abuso de Internet en adolescentes. En E. Echeburúa, F.J. Labrador y E. Becoña (eds.), Adicción a las nuevas tecnologías en adolescentes y jóvenes (pp. 101-128). Madrid: Pirámide.

Figuer, C., González, M., Malo, S. y Casas, F. (2005). El món adolescent en l'entorn de l'ús de I'ordinador i Internet. Perspectiva Escolar, 299, 36-41.

Finn, J. y Lavitt, M. (1994). Computer-based self-help groups for sexual abuse survivors. Social Work With Groups, 17, 21-46.

Fogel, J., Albert, S. M., Schnabel, F., Ditkoff, B. A. y Neugut, A. I. (2002). Internet use and social support in women with breast cancer. Health Psychology, 21, 398-404. 
Fondevila, J.F., Carreras, M. y del Olmo, J.L. (2012). Impacto de las Tecnologías de la Información y la Comunicación en la elección de universidad: el caso de Internet y las redes sociales. EDUTEC, Revista Electrónica de Tecnología Educativa, 39. Recuperado el 10/04/2012.

http://edutec.rediris.es/Revelec2/Revelec39/impacto TIC eleccion universidad internet redes sociales.html

Fowler, J.H. y Christakis, N.A. (2008). Estimating peer effects on health in social networks. Journal of Health Economics, 27, 1386-1391.

Fowler, J.H. y Christakis, N.A. (2009). The Dynamic Spread of Happiness in a Large Social Network: Longitudinal Analysis Over 20 Years in the Framingham Heart Study. British Medical Journal 337, 1-9.

Graner, C., Beranuy, M., Sánchez-Carbonell, X., Chamarro, A. i Castellana, M. (2006) ¿Que uso hacen los jóvenes y adolescentes de internet y del móvil? Comunicación e Ciudadanía, 3, 1-19.

Granovetter, M. S. (1973). The strength of weak ties. American Journal of Sociology, 78, $1360-1380$.

Gross, E.F., Juvonen, J. y Gable, S.L. (2002). Internet use and well-being in adolescence. Journal of Social Issues, 58, 75-90.

Harter, S. (1999). The construction of the self: A developmental perspective. New York: Guilford Press.

Hoybye, M.T., Johansen, C. y Tjornhoj-Thomsen, T. (2005). Online interaction. Effects of storytelling in an Internet breast cancer support group. Psycho-Oncology, 14, 211-220.

Jimenez, L. y Pantoja, A. V. (2007). Autoestima y relaciones Interpersonales en sujetos adictos a Internet. Psicologia-Segunda Epoca, 26, 78- 89.

Katz, J. E. y Aspden, P. (1997). A nation of strangers? Communications of the ACM, 40, 81-86.

King, S.A. y Poulos, S. T. (1998). Using the Internet to treat Generalized Social Phobia and Avoidant Personality Disorder. CyberPsychology and Behavior, 1, 1, 29-36.

Kraut, R., Patterson, M., Landmark, V., Kiesler, S., Mukophadhyay, T. y Scherlis, W. (1998). Internet paradox: a social technology that reduces social involvement and psychological well being? American Psychologist, 53, 1017-1031.

Latiesa, M. (1996).Validez y fiabilidad de las observaciones sociológicas. En M. García, J. Ibáñez y F.Alvira. El análisis de la realidad social. Métodos y técnicas de investigación. Madrid: Alianza.

Lea, M. y Spears, R. (1995). Love at first byte? Building personal relationships over computer networks. En J. T. Wood y S. Duck (Eds.), Under-studied relationships: Off the beaten track (pp. 197-233). Beverly Hills: Sage.

Lenhart, A., Madden, M., Rankin Macgill, A. y Smith, A. (2007). Teens and social media: The use of social media gains a greater foothold in teen life as they embrace the conversational nature of interactive online media. Washington, DC: Pew Internet \& American Life Project. Obtenido el 10 de sepetiembre del 2011. En: 
http://www.pewinternet.org//media//Files/Reports/2007/PIP_Teens_Social_Media_Final.p df

Livingstone, S. (2008). Taking risky opportunities in youthful content creation: teenagers' use of social networking sites for intimacy, privacy and self-expression. New media and society, 10, 393-411.

Losada, J. L. y López-Feal, R. (2003). Métodos de investigación en ciencias humanas y sociales. Madrid: Thomson.

Madara, E., Kalafat, J. y Miller, B. N. (1988). The computerized self-help clearinghouse: Using "high tech" to promote "high touch" support networks. Computers in Human Services, 3, 3953.

Magnuson, M. y Dundes, L. (2008). Gender differences in "Social portraits" reflected in MySpace profiles. Cyberpsychology y Behavior, 11, 239-241.

McKenna, K. y Bargh, J. (1999). Causes and consecuences of social interaction on the Internet: A conceptual framework. Media Psychology, 1, 249-261.

Mesch, G.S. y Talmud, I. (2006). The quality of online and offline relationships: The role of multiplexity and duration of social relationships. The Information Society, 22, 137-148.

Morahan-Martin, J. y Schumacher, P. (2003). Loneliness and social uses of the internet. Computers in Human Behavior, 19, 659-671.

Niemz, K., Griffiths, M. y Banyard, P. (2005). Prevalence of pathological Internet use among university students and correlations with self-esteem, the general health qustionaire (GHQ), and disinhibition. CyberPsychology \& Behavior, 8, 562-570.

Poch, F. (2009). Uso autoinformado de Internet en adolescentes: Perfil psicológico de un uso elevado de red. International Journal of Psychology \& Psychological Therapy, 9, 109-122.

Rapee, R.M. y Heimberg, R.G. (1997). A cognitive-behavioral model of anxiety in social phobia. Behavior Research and Therapy, 35, 741-756.

Raacke, J. y Bonds-Raacke, J. (2008). MySpace and Facebook: Applying the uses and gratifications theory to exploring friend-networking sites. Cyberpsychology \& Behavior, 11, 169-174.

Rivero, G. (2005). Algunas características de la comunicación entre jóvenes y adolescentes en el inicio de relaciones interpersonales en la comunidad. Revista Cubana de Psicologia, 22, 68-70.

Rosenquist, J.N., Fowler, J.H. y Christakis, N.A. (2010). Social Network Determinants of Depression. Molecular Psychiatry 16, 273-281.

Rubio-Gil, A. (2010). Generación digital: patrones de consumo de internet, cultura juvenil y cambio social. Revista de estudios de juventud, 88, 201-221.

Russell, D. W. (1996). UCLA Loneliness scale (version 3): reliability, validity, and factor structure. Journal of Personality Assessment, 66, 20-40.

Sánchez-Carbonell, X., Beranuy, M., Castellana, M., Chamarro, A. y Oberst, U. (2008). La adicción a Internet y al móvil: ¿̇moda o trastorno? Adicciones, 20, 149-160. 
Shaffer, DR (2002). Developmental psychology: Childhood and adolescence. Belmont, CA: Wadsworth.

Selwyn, N. (2003). Apart from technology: understanding peoples, non-use of information and communication technologies in everyday life. Technology in Society, 25, 99-116.

Thayer, S.E. y Ray, S. (2006). Online communication preferences across age, gender, and duration of Internet use. CyberPsychology \& Behavior, 9, 432- 440.

Tully, C.J. (2007). La socialización en el presente digital. Informalización y contextualización. Revista iberoamericana de ciencia, tecnología y sociedad, 8, 9-22.

Yang, C.K., Choe, B.M., Balty, M. y Lee, J.H. (2005). SCL-90-R and 16 PF profiles of senior high school students with excessive Internet use. Canadian Journal of Psychiatry, 50, 407-414.

Young, K. (2009). Online Social Networking: An Australian Perspective. International Journal of Emerging Technologies \& Society, 7, 39-57.

Valkenburg, PM, Peter, J. y Schouten, AP (2006). Friend networking sites and their relationship to adolescents' well-being and social self-esteem. CyberPsychology \& Behavior, 9, 584-590.

Valkenburg, P.M. y Peter, J. (2008). Adolescents' identity experiments on the Internet: Consequences for social competence and self-concept unity. Communication Research, 35, 208-231.

Williams, J. y Solano, C. (1983). The social reality of feelinglonely: friendship and reciprocation. Personality y Social Psychology Bulletin, 9, 237-242.

Zubeidat, I., Salinas, J. y Sierra, J. (2008). Evaluacion de factores asociados a la ansiedad social y a otras psicopatologias en adolescentes, Clinica y salud, 31, 189-196.

Zywica, J. y Danowski, J. (2008). The faces of Facebookers: Investigating social enhancement and social compensation hypotheses; predicting Facebook and offline popularity from sociability and self-esteem, and mapping the meanings of popularity with semantic networks. Journal of Computer-Mediated Communication, 14, 1-34.

\section{Para citar este artículo:}

SURIA, R. (2012). La discapacidad en nuestros jóvenes, ¿ Fomenta las relaciones normalizadas a través de las redes sociales online o les hace adictos a ellas?. EDUTEC, Revista Electrónica de Tecnología Educativa, 41. Recuperado el dd/mm/aa de http://edutec.rediris.es/Revelec2/Revelec41/discapacidad jovenes relaciones redes sociales onlin e.html 


\section{Anexo}

\section{ENCUESTA DE DISCAPACDAD Y UTILIZACIÓN DE REDES SOCIALES DE INTERNET}

\begin{tabular}{|c|c|c|c|c|c|}
\hline \multicolumn{6}{|c|}{ Bloque 1. Perfil sociodemográfico } \\
\hline \multicolumn{6}{|c|}{ 1. ¿Tienes alguna discapacidad? } \\
\hline \multicolumn{3}{|c|}{$\mathrm{Si}$} & No & & \\
\hline \multicolumn{6}{|l|}{ 2. Sexo } \\
\hline \multicolumn{3}{|l|}{ Mujer } & Varón & & \\
\hline \multicolumn{6}{|l|}{ 3. Edad } \\
\hline \multirow{2}{*}{\multicolumn{3}{|c|}{$\begin{array}{l}\text { Menos de } 15 \\
\text { 4. Formación }\end{array}$}} & Entre 26 y 35 & Más de 35 & \\
\hline & & & \multicolumn{3}{|c|}{ 4. Formación } \\
\hline Estudios primarios & \multicolumn{2}{|c|}{ Estudios primarios } & Secundaria & \multicolumn{2}{|c|}{ Universitarios } \\
\hline \multicolumn{6}{|l|}{ 5. Estado civil } \\
\hline \multicolumn{3}{|l|}{ Soltero } & \multicolumn{3}{|l|}{ Pareja } \\
\hline \multicolumn{6}{|c|}{ Bloque 2. Perfil de frecuencia y motivos de utilización de las redes online } \\
\hline \multicolumn{6}{|c|}{ 6. ¿Cuándo te inscribiste en alguna red social? } \\
\hline Antes del 2006 & Antes del 2 & 2008 & Antes del 2010 & Recienten & nente \\
\hline \multicolumn{6}{|c|}{ 7. ¿Entras a alguna? } \\
\hline \multicolumn{3}{|l|}{$\mathrm{Si}$} & No & & \\
\hline \multicolumn{6}{|c|}{ 8. ¿Con qué frecuencia? } \\
\hline 1 vez al mes & Varias veces mes & 1 vez semana & Varias veces semana & 1 vez día & Varias veces día \\
\hline \multicolumn{6}{|c|}{ 9. ¿Cuánto tiempo pasas en la red social? } \\
\hline 5-10 minutos & $10-30$ minutos & $30-60$ minutos & De 1 a 2 horas & a 4 horas & Más de 4 horas \\
\hline \multicolumn{6}{|c|}{ 10. ¿Cuántos amigos tienes en las redes sociales? } \\
\hline De 1 a 5 & De 6 a 10 & Entre 11 y 20 & Entre 21 y 50 & Más de 50 & Más de 100 \\
\hline \multicolumn{6}{|c|}{ 11. ¿Por qué motivo utiliza las redes sociales? } \\
\hline Juegos & Trabajo/estudio & Ligar & Hobbie & Ami & stad \\
\hline 12. Utilizas las & edes sociales para relaci & ionarte con tus am & igos tradicionales & & \\
\hline Nada & Poco & Algo & Bastante & Muc & \\
\hline 13. Utilizas las & edes sociales para relaci & ionarte con tus am & igos de la red & & \\
\hline Nada & Poco & Algo & Bastante & Muc & \\
\hline 14. Utilizas las & edes sociales para relaci & ionarte con tus con & npañeros & & \\
\hline Nada & Poco & Algo & Bastante & Muc & \\
\hline 15. Utilizas las & edes sociales para relaci & ionarte con tus fam & niliares & & \\
\hline Nada & Poco & Algo & Bastante & Muc & \\
\hline & Bloque 3. Percepci & ión de ventajas y re & epercusiones del uso de las & redes online & \\
\hline 16. ¿Es import & nte para ti el número de & amigos que tienes & s en estas redes? & & \\
\hline Nada & Poco & Algo & Bastante & Muc & \\
\hline 17. ¿Crees que & mejoran las relaciones sc & ociales? & & & \\
\hline Nada & Poco & Algo & Bastante & Muc & cho \\
\hline 18. ¿Te han ay & dado a mejoran las relac & ciones sociales? & & & \\
\hline Nada & Poco & Algo & Bastante & Muc & cho \\
\hline 19. ¿Crees que & en general, que las redes & s sociales te facilita & interaccionar con otros? & & \\
\hline Nada & Poco & Algo & Bastante & Muc & cho \\
\hline 20. En las rede & virtuales tienes... & & & & \\
\hline Menos amigos & que en la vida real & Más amigos q & ue en la vida real & Sólo tengo a & amigos en la red \\
\hline 21. ¿Crees que & desde que entras en las & redes de internet $t$ & e has aislado de las amista & des tradiciona & les? \\
\hline Nada & Poco & Algo & Bastante & Muc & cho \\
\hline 22. ¿Crees que & las redes de internet inte & erfieren negativam & ente en tu vida? & & \\
\hline Nada & Poco & Algo & Bastante & Muc & \\
\hline
\end{tabular}

